\title{
Pfaffian hybrid systems
}

\author{
Margarita Korovina $^{1}$ and Nicolai Vorobjov ${ }^{2}$ \\ 1 A.P.Ershov Institute of Informatics Systems, Russian Academy of Sciences \\ korovina@brics.dk, \\ http://www.brics.dk/ korovina \\ 2 Department of Computer Science, University of Bath, Bath BA2 7AY, England \\ nnv@cs.bath.ac.uk, \\ http://www.bath.ac.uk/ masnnv
}

\begin{abstract}
It is well known that in an o-minimal hybrid system the continuous and discrete components can be separated, and therefore the problem of finite bisimulation reduces to the same problem for a transition system associated with a continuous dynamical system. It was recently proved by several authors that under certain natural assumptions such finite bisimulation exists. In the paper we consider o-minimal systems defined by Pfaffian functions, either implicitly (via triangular systems of ordinary differential equations) or explicitly (by means of semi-Pfaffian maps). We give explicit upper bounds on the sizes of bisimulations as functions of formats of initial dynamical systems. We also suggest an algorithm with an elementary (doubly-exponential) upper complexity bound for computing finite bisimulations of these systems.
\end{abstract}

\section{Introduction}

We assume that the reader is familiar with the motivation and basic concepts of the theory of hybrid systems. This material can be found in collection of papers [7]. The more recent accounts are (not exclusively) $[8,3,9]$.

Recall that in certain natural cases continuous and discrete components of a hybrid system can be separated. Moreover, the continuous component allows finite bisimulation, thus reducing the decidability questions for the original system to similar questions for a finite system. An important example having this property is the class of o-minimal hybrid systems, introduced in [9]. The main result of [9] is that under certain natural assumptions, o-minimal systems allow finite bisimulations. This statement was generalized in [3], where a convenient and elementary technique was developed, based on encoding of trajectories of o-minimal dynamical systems in partitioned spaces by means of words in finite alphabets. The elements of bisimulations are then encoded by dotted words.

In the present paper we use the technique of [3] to obtain some quantitative versions of the finite bisimulation theorems. We introduce Pfaffian hybrid systems which essentially reduce to Pfaffian dynamical systems defined by means of equations and inequalities involving Pfaffian functions. The latter are real 
analytic functions satisfying triangular systems of first order partial differential equations with polynomial coefficients. They include polynomials, real algebraic functions, and all major transcendental functions in appropriate domains. Pfaffian functions form the broadest natural class of real analytic functions for whose elements the size or format can be adequately assigned. The concept of the format can be extended to sets in $\mathbb{R}^{n}$ and to maps definable using Pfaffian functions.

We consider dynamical systems defined by Pfaffian functions, either implicitly (via triangular systems of ordinary differential equations) or explicitly (by means of semi-Pfaffian maps). We give explicit upper bounds on the sizes of bisimulations as functions of formats of initial dynamical systems. We also suggest an algorithm with an elementary (doubly-exponential) upper complexity bound for computing finite bisimulations of these systems.

More precisely, the outline of the paper is as follows. In Section 1 we summarize some well-known definitions and results about hybrid systems closely following [3,9]. We also recall the (dotted) words encoding technique from [3]. Section 2 presents a brief digest of Pfaffian functions, upper bounds on topological complexities of semi- and sub-Pfaffian sets, and algorithms for computing their closures and cylindrical cell decompositions. In Section 3 two types of dynamical systems defined using Pfaffian functions are introduced. In Section 4 we consider dynamical systems determined by triangular systems of ordinary differential equations, and prove an upper bound on the size of its bisimulation (note that such systems may not be o-minimal in the sense of $[3,9]$ ). In Section 5 we solve the similar problem for dynamical systems defined by explicit semi-Pfaffian maps. Finally, in Section 6 we propose an algorithm (with the usual for Pfaffian functions theory oracle) which actually computes a finite bisimulation for dynamical systems defined in Section 5 . The complexity of the algorithm is doubly exponential in the format of the input system.

\section{Transition systems and dynamical systems}

In $[3,9]$ it is explained how some central problems in the theory of o-minimal hybrid systems can be reduced to bisimulations of transition systems associated to o-minimal dynamical systems.

The exposition in this section closely follows [3]. The first group of definitions describes transition systems and bisimulations between the transition systems.

Definition 1. Let $Q$ be an arbitrary set and $\rightarrow$ be a binary relation on $Q$. In the context of hybrid systems theory we call $Q$ the set of states, $\rightarrow$ the transition, and $T:=(Q, \rightarrow)$ the transition system.

Definition 2. Given two transition systems $T_{1}:=\left(Q_{1}, \rightarrow_{1}\right)$ and $T_{2}:=\left(Q_{2}, \rightarrow_{2}\right)$ we define a simulation of $T_{1}$ by $T_{2}$ as a binary relation $\sim \subset Q_{1} \times Q_{2}$ such that:

- $\forall q_{1} \in Q_{1} \exists q_{2} \in Q_{2}\left(q_{1} \sim q_{2}\right)$;

- $\forall q_{1}, q_{1}^{\prime} \in Q_{1} \forall q_{2} \in Q_{2} \exists q_{2}^{\prime}\left(\left(q_{1} \sim q_{2} \wedge q_{1} \rightarrow q_{1}^{\prime}\right) \Rightarrow\left(q_{1}^{\prime} \sim q_{2}^{\prime} \wedge q_{2} \rightarrow q_{2}^{\prime}\right)\right)$. 
Definition 3. Abisimulation between two transition systems $T_{1}:=\left(Q_{1}, \rightarrow_{1}\right)$ and $T_{2}:=\left(Q_{2}, \rightarrow_{2}\right)$ is a simulation $\sim \subset Q_{1} \times Q_{1}$ of $T_{1}$ by $T_{2}$ such that the reciprocal relation $\sim^{-1}:=\left\{\left(q_{2}, q_{1}\right) \in Q_{2} \times Q_{1} \mid q_{1} \sim q_{2}\right\}$ is a simulation of $T_{2}$ by $T_{1}$.

Definition 4. A bisimulation between a transition system $T$ and itself is called bisimulation on $T$.

Definition 5. Let $\sim$ be a bisimulation on $T=(Q, \rightarrow)$ and also an equivalence relation on $Q$. Let $\mathcal{P}$ be a partition of $Q$. We say that $\sim$ is a bisimulation with respect to $\mathcal{P}$ if any $P \in \mathcal{P}$ is a union of some equivalence classes of $\sim$.

In this paper we are concerned with estimating cardinality and computing bisimulations in the sense of Definition 5. We now give some definitions concerning dynamical systems.

Definition 6. Let $G_{1} \subset \mathbb{R}^{k_{1}}$ and $G_{2} \subset \mathbb{R}^{k_{2}}$ be open domains. The dynamical system is a map

$$
\gamma: G_{1} \times(-1,1) \rightarrow G_{2} .
$$

In the sequel $\gamma$ will always be definable in an o-minimal structure over $\mathbb{R}$. For a given $\mathbf{x} \in G_{1}$ the set

$$
\Gamma_{\mathbf{x}}=\{\mathbf{y} \mid \exists t \in(-1,1)(\gamma(\mathbf{x}, t)=\mathbf{y})\} \subset G_{2}
$$

is called the trajectory determined by $\mathbf{x}$, and the graph

$$
\widehat{\Gamma}_{\mathbf{x}}=\{(t, \mathbf{y}) \mid \gamma(\mathbf{x}, t)=\mathbf{y}\} \subset(-1,1) \times G_{2}
$$

is called the integral curve determined by $\mathbf{x}$.

Definition 7. The transition system $T_{\gamma}=(Q, \rightarrow)$ associated to the dynamical system $\gamma$ is defined as follows:

- $Q:=G_{2}$, and

- $\mathbf{y}_{1} \rightarrow \mathbf{y}_{2}$ for $\mathbf{y}_{1}, \mathbf{y}_{2} \in Q$ if and only if

$$
\exists \mathbf{x} \in G_{1} \exists t_{1}, t_{2} \in(-1,1)\left(\left(t_{1} \leq t_{2}\right) \wedge\left(\gamma\left(\mathbf{x}, t_{1}\right)=\mathbf{y}_{1}\right) \wedge\left(\gamma\left(\mathbf{x}, t_{2}\right)=\mathbf{y}_{2}\right)\right) .
$$

We now introduce, following [3], a technique of encoding trajectories of dynamical systems by words. Let $\mathcal{P}:=\left\{P_{1}, \ldots, P_{s}\right\}$ be a finite partition of $\gamma\left(G_{1} \times(-1,1)\right)$ definable in the o-minimal struicture. Fix $\mathbf{x} \in G_{1}$. Define the set of points and open intervals in $\mathbb{R}$ :

$\mathcal{F}_{\mathbf{X}}:=\{I \mid I$ is a point or an interval in $(-1,1)$ maximal w.r.t. inclusion for the

$$
\text { property } \left.\exists i \in\{1, \ldots, s\} \forall t \in I\left(\gamma(\mathbf{x}, t) \in P_{i}\right)\right\} \text {. }
$$

Let the cardinality $\left|\mathcal{F}_{\mathbf{x}}\right|=r$ and $y_{1}<\cdots<y_{r}$ be the set of representatives of $\mathcal{F}_{\mathbf{x}}$ such that $\gamma\left(\mathbf{x}, y_{j}\right) \in P_{i_{j}}$. Then define the word $\omega:=P_{i_{1}} \cdots P_{i_{r}}$ in alphabet 
$\mathcal{P}$. Informally, $\omega$ is the list of names of elements of the partition in the order they are visited by trajectory $\Gamma_{\mathbf{x}}$.

Let $\mathbf{y} \in \Gamma_{\mathbf{x}}$. Then $\mathbf{y} \in P_{i_{j}}$ for some $1 \leq j \leq r$, where $P_{i_{j}}$ is a letter in $\omega$. We represent the location of $\mathbf{y}$ on trajectory $\Gamma_{\mathbf{x}}$ by the dotted word

$$
\dot{\omega}:=P_{i_{1}} \cdots \dot{P}_{i_{j}} \cdots P_{i_{r}}
$$

It will be convenient to use the operation

$$
\operatorname{undot}(\dot{\omega})=\omega:=P_{i_{1}} \cdots P_{i_{j}} \cdots P_{i_{r}} .
$$

In the sequel we will always assume that a dynamical systems $\gamma$ is injective. In this case there is a unique dotted word associated to a given $\mathbf{y} \in \gamma\left(G_{1} \times(-1,1)\right)$.

Introduce sets of words $\Omega:=\left\{\omega \mid \mathrm{x} \in G_{1}\right\}, \dot{\Omega}:=\left\{\dot{\omega} \mid \mathrm{x} \in G_{1}\right\}$.

The following statement is an easy consequence of o-minimality.

Lemma 1. [3] The set $\Omega$ is finite.

An obvious (purely combinatorial) corollary is that $\dot{\Omega}$ is also finite.

Definition 8. The transition system $T_{\dot{\Omega}}$ is defined as follows:

- $Q:=\dot{\Omega}$, and

- $\dot{\omega}_{1} \rightarrow \dot{\omega}_{2}$ for $\dot{\omega}_{1}, \dot{\omega}_{2} \in Q$ if and only if $\omega_{1}=\omega_{2}$ and the dot on $\dot{\omega}_{2}$ is on the righter (or the same) position than the dot on $\dot{\omega}_{1}$.

Theorem 1. [3] Let the o-minimal dynamical system $\gamma$ be bijective, and the partition $\mathcal{P}$ be definable in the o-minimal structure. Then there is a finite bisimulation on $T_{\gamma}$ with respect to $\mathcal{P}$.

Proof. To prove the theorem one first shows that $T_{\dot{\Omega}}$ is a bisimulation of $T_{\gamma}$, and then considers the following equivalence relation $\sim$ on $G_{2}: \mathbf{y}_{1} \sim \mathbf{y}_{2}$ iff for respective pre-images $\left(\mathbf{x}_{1}, t_{1}\right),\left(\mathbf{x}_{2}, t_{2}\right)$, the locations of $\mathbf{y}_{1}, \mathbf{y}_{2}$ on trajectories $\Gamma_{\mathbf{x}_{1}}, \Gamma_{\mathbf{x}_{2}}$ are described by the same dotted word $\dot{\omega}$. Then $\sim$ is the required bisimulation (see details in [3]).

\section{Pfaffian functions and related sets}

This section is a digest of the theory of Pfaffian functions and sets definable with Pfaffian functions. The detailed exposition can be found in the survey [4].

Definition 9. A Pfaffian chain of the order $r \geq 0$ and degree $\alpha \geq 1$ in an open domain $G \subset \mathbb{R}^{n}$ is a sequence of real analytic functions $f_{1}, \ldots, f_{r}$ in $G$ satisfying differential equations

$$
\frac{\partial f_{j}}{\partial x_{i}}=g_{i j}\left(\mathbf{x}, f_{1}(\mathbf{x}), \ldots, f_{j}(\mathbf{x})\right)
$$


for $1 \leq j \leq r, 1 \leq i \leq n$. Here $g_{i j}\left(\mathbf{x}, y_{1}, \ldots, y_{j}\right)$ are polynomials in $\mathbf{x}=$ $\left(x_{1}, \ldots, x_{n}\right), y_{1}, \ldots, y_{j}$ of degrees not exceeding $\alpha$. A function

$$
f(\mathbf{x})=P\left(\mathbf{x}, f_{1}(\mathbf{x}), \ldots, f_{r}(\mathbf{x})\right),
$$

where $P\left(\mathbf{x}, y_{1}, \ldots, y_{r}\right)$ is a polynomial of a degree not exceeding $\beta \geq 1$, is called $a$ Pfaffian function of order $r$ and degree $(\alpha, \beta)$.

Apart from polynomials, the class of Pfaffian functions includes real algebraic functions, exponentials, logarithms, trigonometric functions, their compositions, and other major transcendental functions in appropriate domains (see [4]).

Definition 10. A set $X \subset \mathbb{R}^{n}$ is called semi-Pfaffian in an open domain $G \subset$ $\mathbb{R}^{n}$ if it consists of points in $G$ satisfying a Boolean combination of some atomic equations and inequalities $f=0, g>0$, where $f, g$ are Pfaffian functions having a common Pfaffian chain defined in $G$. A semi-Pfaffian set $X$ is restricted in $G$ if its topological closure lies in $G$.

Definition 11. A set $X \subset \mathbb{R}^{n}$ is called sub-Pfaffian in an open domain $G \subset \mathbb{R}^{n}$ if it is an image of semi-Pfaffian set under a projection into a subspace.

In the sequel we will be dealing with the following subclass of sub-Pfaffian sets.

Definition 12. Consider the closed cube $[-1,1]^{m+n}$ in an open domain $G \subset$ $\mathbb{R}^{m+n}$ and the projection map $\pi: \mathbb{R}^{m+n} \rightarrow \mathbb{R}^{n}$. A subset $Y \subset[-1,1]^{n}$ is called restricted sub-Pfaffian if $Y=\pi(X)$ for a restricted semi-Pfaffian set $X \subset$ $[-1,1]^{m+n}$.

Note that a restricted sub-Pfaffian set need not be semi-Pfaffian.

Definition 13. Consider a semi-Pfaffian set

$$
X:=\bigcup_{1 \leq i \leq M}\left\{\mathbf{x} \in \mathbb{R}^{s} \mid f_{i 1}=\cdots=f_{i I_{i}}, g_{i 1}>0, \ldots, g_{i J_{i}}>0\right\} \subset G,
$$

where $f_{i j}, g_{i j}$ are Pfaffian functions with a common Pfaffian chain of order $r$ and degree $(\alpha, \beta)$, defined in an open domain $G$. Its format is a tuple $(r, N, \alpha, \beta, s)$, where $N \geq \sum_{1 \leq i \leq M}\left(I_{i}+J_{i}\right)$. For $s=m+n$ and a sub-Pfaffian set $Y \subset \mathbb{R}^{n}$ such that $Y=\pi(\bar{X})$, its format is the format of $X$.

We will refer to the representation of a semi-Pfaffian set in the form (2) as to disjunctive normal form (DNF).

Remark 1. In this paper we are concerned with upper bounds on sizes of bisimulations and complexities of computations, as functions of the format. In the case of Pfaffian dynamical systems these sizes and complexities also depend on the domain $G$. So far our definitions imposed no restrictions on an open set $G$, thus allowing it to by arbitrarily complex and induce this complexity on the corresponding semi- and sub-Pfaffian sets. To avoid this we will always assume in the context of Pfaffian dynamical systems that $G$ is "simple", like $\mathbb{R}^{n}$, or $(-1,1)^{n}$. 
Theorem 2. [6,11] Consider a semi-Pfaffian set $X \subset G \subset \mathbb{R}^{n}$, where $G$ is an open domain, represented in DNF with format $(r, N, \alpha, \beta, n)$. Then the sum of the Betti numbers (in particular, the number of connected components) of $X$ does not exceed

$$
N^{n} 2^{r(r-1) / 2} O(n \beta+\min \{n, r\} \alpha)^{n+r} .
$$

In this paper we examine complexities of algorithms for computing bisimulations. In order to estimate the "efficiency" of a computation we need to specify more precisely a model of computation. As such we use a real numbers machine which is an analogy of a classical Turing machine but allows the exact arithmetic and comparisons on real numbers. Since we are interested only in upper complexity bounds for algorithms, we have no need in a formal definition of this model of computation (it can be found in [2]). In some of our computational problems we will need to modify the standard real numbers machine by equipping it with an oracle for deciding feasibility of any system of Pfaffian equations and inequalities. An oracle is a subroutine which can be used by a given algorithm any time the latter needs to check feasibility. We assume that this procedure always gives a correct answer ("true" or "false") though we do not specify how it actually works. An elementary step of a real numbers machine is either an arithmetic operation, or a comparison (branching) operation, or an oracle call. The complexity of a real numbers machine is the number of elementary steps it makes in worst case until termination, as a function of the format of the input.

Now we define cylindrical decompositions of semi- and sub-Pfaffian sets.

Definition 14. Cylindrical cell in $[-1,1]^{n}$ is defined by induction as follows.

1. Cylindrical 0 -cell in $[-1,1]^{n}$ is an isolated point.

2. Cylindrical 1-cell in $[-1,1]$ is an open interval $(a, b) \subset[-1,1]$.

3. For $n \geq 2$ and $0 \leq k<n$ a cylindrical $(k+1)$-cell in $[-1,1]^{n}$ is either a graph of a continuous bounded function $f: C \rightarrow \mathbb{R}$, where $C$ is a cylindrical $(k+1)$-cell in $[-1,1]^{n-1}$, or else a set of the form

$$
\begin{gathered}
\left\{\left(x_{1}, \ldots, x_{n}\right) \in[-1,1]^{n} \mid\left(x_{1}, \ldots, x_{n-1}\right) \in C\right. \text { and } \\
\left.f\left(x_{1}, \ldots, x_{n-1}\right)<x_{n}<g\left(x_{1}, \ldots, x_{n-1}\right)\right\},
\end{gathered}
$$

where $C$ is a cylindrical $k$-cell in $[-1,1]^{n-1}$, and $f, g: C \rightarrow[-1,1]$ are continuous bounded functions such that $f\left(x_{1}, \ldots, x_{n-1}\right)<g\left(x_{1}, \ldots, x_{n-1}\right)$ for all points $\left(x_{1}, \ldots, x_{n-1}\right) \in C$.

Definition 15. Cylindrical cell decomposition $\mathcal{D}$ of a subset $A \subset[-1,1]^{n}$ is defined by induction as follows.

1. If $n=1$, then $\mathcal{D}$ is a finite family of pair-wise disjoint cylindrical cells (i.e., isolated points and intervals) whose union is $A$.

2. If $n \geq 2$, then $\mathcal{D}$ is a finite family of pair-wise disjoint cylindrical cells in $[-1,1]^{n}$ whose union is $A$ and there is a cylindrical cell decomposition of $\pi(A)$ such that $\pi(C)$ is its cell for each $C \in \mathcal{D}$, where $\pi: \mathbb{R}^{n} \rightarrow \mathbb{R}^{n-1}$ is the projection map onto the coordinate subspace of $x_{1}, \ldots, x_{n-1}$. 
Definition 16. Let $B \subset A \subset[-1,1]^{n}$ and $\mathcal{D}$ be a cylindrical cell decomposition of $A$. Then $\mathcal{D}$ is compatible with $B$ if for any $C \in \mathcal{D}$ we have either $C \subset B$ or $C \cap B=\emptyset$ (i.e., some subset $\mathcal{D}^{\prime} \subset \mathcal{D}$ is a cylindrical cell decomposition of $B$ ).

Definition 17. For a given finite family $f_{1}, \ldots, f_{N}$ of Pfaffian functions $f_{i}$ in an open domain $G$ define its consistent sign assignment as a non-empty semiPfaffian set in $G$ of the kind

$\left\{\mathbf{x} \in G \mid f_{i_{1}}=\cdots=f_{i_{N_{1}}}=0, f_{i_{N_{1}}+1}>0 \ldots, f_{i_{N_{2}}}>0, f_{i_{N_{2}}+1}<0, \ldots, f_{i_{N}}<0\right\}$, where $i_{1}, \ldots, i_{N_{1}}, \ldots, i_{N_{2}}, \ldots, i_{N}$ is a permutation of $1, \ldots, N$.

Theorem 3. $[5,10]$ Let $f_{1}, \ldots, f_{N}$ be a family of Pfaffian functions in an open domain $G \subset \mathbb{R}^{s}, G \supset[-1,1]^{s}$ having a common Pfaffian chain of order $r$, and degrees $(\alpha, \beta)$. Then there is an algorithm (with the oracle) producing a cylindrical cell decomposition of $[-1,1]^{\text {s }}$ which is compatible with each consistent sign assignment of $f_{1}, \ldots, f_{N}$. Each cell is a sub-Pfaffian set represented as a projection of a semi-Pfaffian set in DNF. The number of cells, the components of their formats and the complexity of the algorithm are less than

$$
(\alpha+\beta N)^{r^{O(n)} 2^{O\left(n^{2}\right)}} .
$$

\section{Pfaffian dynamical systems}

Definition 18. A triangular system of ordinary differential equations is defined by

$$
\dot{\mathbf{x}}=\mathbf{f}(t, \mathbf{x}),
$$

where $\mathbf{x} \in \mathbb{R}^{n}$, and $\mathbf{f}$ is a vector-function $\mathbf{f}=\left(f_{1}, \ldots, f_{n}\right)$ where

$$
f_{i} \in \mathbb{R}\left[t, x_{1}, x_{2}, \ldots, x_{i}\right]
$$

for every $1 \leq i \leq n$. By a solution of (3) with initial conditions $\left(t_{0}, \mathbf{x}_{0}\right)$, $t_{0} \in(-1,1)$ we mean any analytic vector-function $\varphi:(-1,1) \rightarrow \mathbb{R}^{n}$ such that $d \varphi / d t=\mathbf{f}(t, \varphi)$ for all $t \in(-1,1)$ and $\varphi\left(t_{0}\right)=\mathbf{x}_{0}$.

From the Definition 9 it follows that any solution of (3) is a vector of Pfaffian functions in the common domain $(-1,1)$.

To any system (3) we can relate a dynamical system $\gamma: G \times(-1,1) \rightarrow G$, where $G=I_{1} \times \cdots \times I_{n}$, and $I_{i}$ is an open interval (possibly unbounded) for all $1 \leq i \leq n$. More precisely, assume that for any $\mathbf{x} \in G$ the system has a solution $\varphi$ with initial conditions $(\mathbf{x}, 0)$. Then $\gamma(\mathbf{x}, t):=\varphi(t)$.

Along with the dynamical systems associated with triangular systems of the kind (3) we will consider Pfaffian dynamical systems defined as follows.

Definition 19. A dynamical system

$$
\gamma: G \times(-1,1) \rightarrow G
$$

where $G$ is open in $\mathbb{R}^{n}$ and $\gamma$ is a map with a semi-Pfaffian graph, is called Pfaffian dynamical system. 
Remark 2. Observe that the dynamical system $\gamma$ associated with (3) may not be a Pfaffian dynamical system in the sense of the last definition since $\gamma$, being a Pfaffian vector-function for any fixed $\mathbf{x}$, is not necessarily a Pfaffian map for variable $\mathbf{x}$.

\section{Bisimulations of dynamical systems associated with a differential equation}

Let in the system (3) the degree $\operatorname{deg}\left(f_{i}\right)<\alpha$ for any $1 \leq i \leq n$ and the associated dynamical system $\gamma$ be bijective. Let $T=(G, \rightarrow)$ be the transition system associated with $\gamma$. Consider a partition $\mathcal{P}:=\left\{P_{1}, \ldots, P_{s}\right\}$ of $G$ into $s$ semi-Pfaffian sets $P_{j}$ each having the format $(r, N, \alpha, \beta, n)$. Let $m:=\max \{n, r\}$, $M:=\max \{n, N\}$.

Theorem 4. There is a bisimulation on $T$ with respect to $\mathcal{P}$ consisting of

$$
s^{s M^{n} 2^{m(m-1) / 2} O(n(\alpha+\beta))^{n+m}}
$$

equivalence classes.

Proof. We use the notations and arguments of Section 1. First we estimate the length $\ell$ of the word $\omega$ for any $\mathbf{x} \in G$. Fix $\mathbf{x}$. Since $\ell$ coincides with the total number of connected components of intersections

$$
P_{j} \cap \Gamma_{\mathbf{x}}=\left\{\mathbf{y} \mid \exists t \in(-1,1)\left(\mathbf{y}=\gamma(\mathbf{x}, t) \wedge \mathbf{y} \in P_{j}\right)\right\}
$$

for all $1 \leq j \leq s$. The semi-Pfaffian set $\widehat{P}_{j}:=\left\{(\mathbf{y}, t) \mid \mathbf{y}=\gamma(\mathbf{x}, t) \wedge \mathbf{y} \in P_{j}\right\}$ has the format $(m, M, \alpha, \beta, n)$, thus according to Theorem 2 the number of its connected components does not exceed

$$
L:=M^{n} 2^{m(m-1) / 2} O(n(\alpha+\beta))^{n+m} .
$$

Since $P_{j} \cap \Gamma_{\mathbf{x}}$ is the projection of $\widehat{P}_{j}$ along $t$, the number of all connected components of $P_{j} \cap \Gamma_{x}$ is also less or equal to $L$, and the total number of connected components for all $1 \leq j \leq s$ does not exceed $s L$. Since the number of distinct letters in any word $\omega$ is at most $s$, the number of all words in the set $\Omega$ does not exceed (4). Then the cardinality of the set $\dot{\Omega}$ of all dotted words also does not exceed (4). It remains to notice that due to Theorem 1 , the finite transition system $T_{\dot{\Omega}}$ is a bisimulation of $T$.

\section{Bisimulations of Pfaffian dynamical systems}

Consider a bijective Pfaffian dynamical system $\gamma: G \times(-1,1) \rightarrow G$, where $G=(-1,1)^{n}$, and a partition $\mathcal{P}:=\left\{P_{1}, \ldots, P_{s}\right\}$ of $G$ into $s$ semi-Pfaffian sets $P_{j}$. Let the graph of $\gamma$ and each set $P_{j}$ have the format $(r, N, \alpha, \beta, n)$, and all Pfaffian functions involved have the common Pfaffian chain. 
Theorem 5. There is a bisimulation on $T$ with respect to $\mathcal{P}$ consisting of

$$
s^{s N^{n} 2^{r(r-1) / 2} O(n(\alpha+\beta))^{n+r}}
$$

equivalence classes.

Proof. A straightforward adjustment of the proof of Theorem 4.

Using the Pfaffian dependence on $\mathbf{x}$ in the case of a Pfaffian dynamical system we obtain another upper bound on the size of the bisimulation which is asymptotically better in general than the bound in Theorem 5 (except the case when $n$ is significantly larger than the rest of the parameters).

Theorem 6. There is a bisimulation on $T$ with respect to $\mathcal{P}$ consisting of

$$
(\alpha+\beta s N)^{r^{O(n)} 2^{O\left(n^{2}\right)}}
$$

equivalence classes.

Proof. Consider the family of Pfaffian functions in the domain

$$
G \times(-1,1) \times G
$$

consisting of all functions in variables $\mathbf{x}, t, \mathbf{y}$ involved in the defining formulae for the graph of the map $\gamma:(\mathbf{x}, t) \mapsto \mathbf{y}$, and for all sets $P_{j}$ considered in the latter case as functions in variables $\mathbf{y}$. According to Theorem 3 , there is a cylindrical decomposition $\mathcal{D}$ for this family with respect to $(\mathbf{x}, t, \mathbf{y})$, consisting of at most (6) cylindrical cells. By the definition of cylindrical decomposition, $\mathcal{D}$ induces the cylindrical decomposition on $G$ (equipped with coordinates $\mathbf{x}$ ) which we denote by $\mathcal{E}$.

We claim that for any cell $C \in \mathcal{E}$ and any two points $\mathbf{x}_{1}, \mathbf{x}_{2} \in C$ the trajectories $\Gamma_{\mathbf{x}_{1}}, \Gamma_{\mathbf{x}_{2}} \in G$ are intersecting sets $P_{1}, \ldots, P_{s}$ in the same order (i.e., are encoded by the same word from $\Omega$ ). Indeed, let $\pi: G \times(-1,1) \times G \rightarrow G$ be the projection on $G$ with coordinates x. Decomposition $\mathcal{D}$ induces cylindrical decompositions $\mathcal{D}_{1}$ and $\mathcal{D}_{2}$ on $\pi^{-1}\left(\mathbf{x}_{1}\right)$ and $\pi^{-1}\left(\mathbf{x}_{2}\right)$ respectively. In particular, each of the integral curves $\widehat{\Gamma}_{\mathbf{x}_{1}}$ and $\widehat{\Gamma}_{\mathbf{x}_{2}}$ is decomposed into a sequence of alternating points and open intervals. Due to basic properties of a cylindrical decomposition, there is a natural bijection $\psi: \mathcal{D}_{1} \rightarrow \mathcal{D}_{2}$ such that

(i) the restriction of $\psi$ to the set of all cells in $\widehat{\Gamma}_{\mathbf{x}_{1}}$ is a bijection onto the set of all cells in $\widehat{\Gamma}_{\mathbf{x}_{2}}$

(ii) for each $1 \leq j \leq s$ the restriction of $\psi$ to the set of all cells in $(-1,1) \times P_{j} \subset$ $\pi^{-1}\left(\mathbf{x}_{1}\right)$ is a bijection onto the set of all cells in $(-1,1) \times P_{j} \subset \pi^{-1}\left(\mathbf{x}_{2}\right)$.

It follows that if a cell $B \in \mathcal{D}_{1}$ and $B \subset \widehat{\Gamma}_{\mathbf{x}_{1}} \cap\left((-1,1) \times P_{j}\right)$ for some $1 \leq j \leq s$, then $\psi(B) \subset \widehat{\Gamma}_{\mathbf{x}_{2}} \cap\left((-1,1) \times P_{j}\right)$. The claim is proved.

It follows that the cardinality of $\Omega$ does not exceed the cardinality of $\mathcal{E}$ which does not exceed the cardinality of $\mathcal{D}$ which in turn is at most (6). Therefore, the cardinality of $\dot{\Omega}$ does not exceed (6), and the theorem is proved. 


\section{Computing bisimulations}

In this section we introduce an algorithm for computing finite bisimulations described in Theorem 6 . It is sufficient to construct the set of dotted words $\dot{\Omega}$ corresponding to the bijective Pfaffian dynamical system $\gamma: G \times(-1,1) \rightarrow G$ (with $G=(-1,1)^{n}$ ) and a partition $\mathcal{P}:=\left\{P_{1}, \ldots, P_{s}\right\}$. Since $\dot{\Omega}$ is trivially obtained from the set $\Omega$, we will be constructing the latter set.

The algorithm applies the procedure from Theorem 3 to the family of Pfaffian functions consisting of all functions in variables $\mathbf{x}, t, \mathbf{y}$ involved in the defining formulae for the graph of the map $\gamma:(\mathbf{x}, t) \mapsto \mathbf{y}$, and for all sets $P_{j}$ considered in the latter case as functions in variables $\mathbf{y}$. As a result, the algorithm produces a cell decomposition $\mathcal{D}$ which induces the cell decomposition $\mathcal{E}$ (see the proof of Theorem 6). Using the oracle, the algorithm selects the cells from $\mathcal{D}$ which are subsets of $\{(\mathbf{x}, t, \mathbf{y}) \mid \mathbf{y}=\gamma(\mathbf{x}, t\}$. Denote the set of the selected cells by $\mathcal{B}$. Observe that for any fixed $\mathbf{x}^{\prime} \in G$ the set $\bigcup_{B \in \mathcal{B}} B \cap\left\{\mathbf{x} \mid \mathbf{x}=\mathbf{x}^{\prime}\right\}$ coincides with the integral curve $\widehat{\Gamma}_{\mathbf{x}^{\prime}}$. Then the algorithm determines the order in which the cells $B \in \mathcal{B}$ intersected with $\left\{\mathbf{x} \mid \mathbf{x}=\mathbf{x}^{\prime}\right\}$ appear in the trajectory $\Gamma_{\mathbf{x}^{\prime}}$.

More precisely, for each pair of distinct cells $B_{1}, B_{2} \in \mathcal{B}$ the algorithm decides, using the oracle, whether

$$
\exists \mathbf{x} \exists t_{1} \exists t_{2} \exists \mathbf{y}_{1} \exists \mathbf{y}_{2}\left(\left(\mathbf{x}, t_{1}, \mathbf{y}_{1}\right) \in B_{1} \wedge\left(\mathbf{x}, t_{2}, \mathbf{y}_{2}\right) \in B_{2} \wedge\left(t_{1}<t_{2}\right)\right) .
$$

For a given $C \in \mathcal{E}$, after all pairs of cells are processed we get the ordered set of cells $B_{1}, \ldots, B_{k}$ in $\mathcal{D}$ such that for any $1 \leq i \leq k$ and any $\mathbf{x}^{\prime} \in C$ the sequence of points and intervals

$$
B_{1} \cap\left\{\mathbf{x} \mid \mathbf{x}=\mathbf{x}^{\prime}\right\}, \ldots, B_{k} \cap\left\{\mathbf{x} \mid \mathbf{x}=\mathbf{x}^{\prime}\right\}
$$

forms the integral curve $\widehat{\Gamma}_{\mathbf{x}^{\prime}}$. By the definition of the cylindrical decomposition, for any pair $B_{i}, P_{j}$ either $B_{i} \subset\left(C \times(-1,1) \times P_{j}\right)$ or $B_{i} \cap\left(C \times(-1,1) \times P_{j}\right)=\emptyset$. The algorithm uses the oracle to decide for every pair which of these two cases takes place. As the result, the sequence $B_{1}, \ldots, B_{k}$ becomes partitioned into subsequences of the kind

$$
\left(B_{1}, \ldots, B_{k_{1}}\right),\left(B_{k_{1}+1}, \ldots, B_{k_{2}}\right), \ldots,\left(B_{k_{\ell-1}+1}, \ldots, B_{k}\right)
$$

where for any $i, 0 \leq i \leq \ell-1$, the cells $B_{k_{i}+1}, \ldots, B_{k_{i+1}}$ lie in $C \times(-1,1) \times P_{j_{i}}$ for some $j_{i}$, while $B_{k_{i}} \cap C \times(-1,1) \times P_{j_{i}}=\emptyset$ and $B_{k_{i+1}+1} \cap C \times(-1,1) \times P_{j_{i}}=\emptyset$. Then the word $\omega:=P_{j_{0}} \cdots P_{j_{\ell-1}}$ corresponds to the cell $C$. Considering all cells in $\mathcal{E}$ the algorithm finds $\Omega$ and then $\dot{\Omega}$. This completes the description of the construction of $T_{\dot{\Omega}}$. It remains to construct the bisimulation $\sim$ on $G$.

As it was explained in the proof of Theorem 1 , for $\mathbf{y}_{1}, \mathbf{y}_{2} \in G$ we have $\mathbf{y}_{1} \sim \mathbf{y}_{2}$ iff for respective pre-images $\left(\mathbf{x}_{1}, t_{1}\right),\left(\mathbf{x}_{2}, t_{2}\right)$, the locations of $\mathbf{y}_{1}, \mathbf{y}_{2}$ on trajectories $\Gamma_{\mathbf{x}_{1}}, \Gamma_{\mathbf{x}_{2}}$ are described by the same dotted word. Fix a cell $C \in \mathcal{E}$. To all points $\mathbf{x} \in C$ correspond the trajectories $\Gamma_{\mathbf{x}}$ encoded by the same word, say $\omega:=P_{i_{0}} \cdots P_{i_{q}} \cdots P_{i_{\ell-1}}$. Consider a dotted word $\dot{\omega}:=P_{i_{0}} \cdots \dot{P}_{i_{q}} \cdots P_{i_{\ell-1}}$. 
Then all points $\mathbf{y} \in \Gamma_{\mathbf{x}}$ (for various $\mathbf{x} \in C$ ) whose locations are described by $\dot{\omega}$ form the set

$$
A_{\dot{\omega}}(C):=\left\{\mathbf{y} \in G \mid \exists \mathbf{x} \in C \exists t\left((\mathbf{x}, t, \mathbf{y}) \in\left(B_{k_{q}+1} \cup \cdots \cup B_{k_{q+1}}\right)\right)\right\} .
$$

Notice that $A_{\dot{\omega}}(C)$ is a sub-Pfaffian set with components of the format not exceeding (6). The equivalence relation $\sim$ is now defined by the partition

$$
G=\bigcup_{\dot{\omega} \in \dot{\Omega}} A_{\dot{\omega}}
$$

into disjoint classes

$$
A_{\dot{\omega}}:=\bigcup_{C \in \mathcal{E}} A_{\dot{\omega}}(C)
$$

This completes the description of the algorithm.

A straightforward analysis shows that the complexity of the algorithm does not exceed (6), taking into account the bounds from Theorem 3 .

\section{$7 \quad$ Future research}

Observe that upper bounds from Theorems 5 and 6 on the size of bisimulations are doubly exponential in some parameters of the format of the original dynamical system. It looks feasible that there exists a singly exponential upper bound. The proof would require avoiding cylindrical cell decomposition technique which is intrinsically doubly exponential. Instead, it could use ideas related to those employed in effective quantifier elimination over real closed fields (see, e.g., [1]) and in recent upper bounds on topological complexity of definable sets [6].

\section{Acknowledgements}

The first author was supported in part by Grant Scientific School 2112.2003.1 and by the London Mathematical Society scheme 2 grant. A part of the work on this paper was done while she visited the Department of Computing and Software of McMaster University in Spring 2004. The second author was supported by the European RTN Network RAAG 2002-2006 (contract HPRN-CT-2001-00271).

\section{References}

1. S. Basu, R. Pollack and M.-F. Roy, Algorithms in Real Algebraic Geometry, Springer, Berlin-Heidelberg, 2003.

2. L. Blum, , F. Cucker, M. Shub, and S. Smale, Complexity and Real Computation, Springer, New York, 1997.

3. T. Brihaye, C. Michaux, C. Riviere, C. Troestler, On o-minimal hybrid systems, in: Hybrid Systems: Computation and Control, R. Alur, G. J. Pappas, (Eds.), LNCS, 2993, Springer, Heidelberg, 2004, 219-233. 
4. A. Gabrielov, N. Vorobjov, Complexity of computations with Pfaffian and Noetherian functions, in: Normal Forms, Bifurcations and Finiteness Problems in Differential Equations, Yu. Ilyashenko et al., (Eds.), NATO Science Series II, 137, Kluwer, 2004, 211-250.

5. A. Gabrielov, N. Vorobjov, Complexity of cylindrical decompositions of subPfaffian sets, J. Pure and Appl. Algebra, 164, 1-2, 2001, 179-197.

6. A. Gabrielov, N. Vorobjov, Betti numbers of semialgebraic sets defined by quantifier-free formulae, to appear in: Discrete and Computational Geometry, 2004.

7. R.L. Grossman, et al., (Eds.), Hybrid Systems, LNCS, 736, Springer, BerlinHeidelberg, 1993, $474 \mathrm{p}$.

8. T. A. Henzinger, The theory of hybrid automata, in: Proceedings of 11th Ann. Symp. Logic in Computer Sci., IEEE Press, 1996, 278-292.

9. G. Lafferriere, G.J. Pappas, S. Sastry, O-minimal hybrid systems, Math. Control Signals Systems, 13, 2000, 1-21.

10. S. Pericleous, N. Vorobjov, New complexity bounds for cylindrical decompositions of sub-Pfaffian sets, in: Discrete and Computational Geometry. Goodman-Pollack Festschrift, B. Aronov et al. (Eds.), Springer, 2003, 673-694.

11. T. Zell, Betti numbers of semi-Pfaffian sets, J. Pure Appl. Algebra, 139, 1999, 323-338. 